Check for updates

Cite this: RSC Adv., 2017, 7, 42462

\title{
Highly efficient MOF-based self-propelled micromotors for water purification $\uparrow$
}

\begin{abstract}
Ruiqin Wang, Weilin Guo, (D) * Xianghui Li, Zhonghua Liu, Hua Liu and Shiyang Ding
Self-propelled metal-organic framework (MOF)-based Janus micromotors that propel autonomously in hydrogen peroxide and display effective remediation of contaminated water is presented in this work. The novel Janus micromotors rely on the asymmetric deposition of a catalytically active Ag patch on the surface of MOF composite microspheres. The active Ag sites are used for the splitting of $\mathrm{H}_{2} \mathrm{O}_{2}$ to form oxygen bubbles. As a result, these Janus micromotors can reach a high speed of over $310 \mu \mathrm{m} \mathrm{s}^{-1}$ due to effective bubble propulsion, which is comparable to common Pt-based micromotors. By coupling the high catalytic capacity of MOFs with their autonomous propulsion, the MOF-based micromotors are shown to play a dominant role in the effective removal of organic pollutants. In addition, scanning electronic microscopy, Fourier-transform infrared spectroscopy and energy dispersive X-ray spectroscopy are performed to verify their morphology and composition. Based on the obtained results, a potential mechanism of the motion and the high catalytic activity is also proposed. It is expected that these energy saving micromotors with catalytic activity should be unprecedentedly spread in real applications.
\end{abstract}

Received 23rd July 2017

Accepted 29th August 2017

DOI: $10.1039 / \mathrm{c} 7 \mathrm{ra0} 8127 \mathrm{~h}$

rsc.li/rsc-advances resources discovery. ${ }^{4,5}$ In particular, these autonomously moving micromotors have been shown recently to be extremely useful for imparting significant mixing (without external forced convection, e.g., stirring) during water-purification. Therefore, fabrication of new effective micromotors that are capable of removing various pollutants could have major impacts upon public safety.

Metal-organic frameworks (MOFs) are emerging as the most promising stable catalysts from this family owing to their rationally designed framework structure, tunable pore size of the order of molecular dimension, and chemical tailoring of the inner surface of the channels and cavities. ${ }^{6,7}$ Another important feature is that most of the metal cations can participate in MOF formation compared with inorganic materials which are based on a few cations such as $\mathrm{Si}, \mathrm{Al}$, and $\mathrm{P}^{8}$ Thanks to their unique and attractive features, MOFs have contributed to developments in the environmental remediation field concerning adsorption ${ }^{9}$ and catalysis. ${ }^{10}$ Zeolitic imidazolate frameworks (ZIFs) as a family of MOFs with excellent thermal and chemical stabilities are synthesized by coordinating transition metals $\left(\mathrm{Zn}^{2+}, \mathrm{Co}^{2+}\right)$ with imidazole ligands. ${ }^{11-14}$ Fortunately, it is very interesting to find that iron-based ZIFs system possesses active sites (Fe ions) for heterogeneous Fenton reaction with superb catalytic activity. ${ }^{15}$

Therefore, given the stability and high catalytic activity of Febased ZIFs materials, firstly, we demonstrated the assembly of ZIF-Zn-Fe (with the catalytically active $\mathrm{Fe}^{2+}$ incorporated) on the surface of micron-sized polystyrene spherical particles. Subsequently, the multifunctional Janus micromotors are made
School of Resources and Environment,
China. E-mail: Chm_guowl@ujn.edu.cn

$\dagger$ Electronic supplementary information (ESI) available. See DOI: 10.1039/c7ra08127h 
up of a catalytic Ag layer hemisphere placed on the surface of polystyrene@ZIF-Zn-Fe core-shell microparticles, combining the remarkable adsorption and high catalytic activity for the generation of ${ }^{\circ} \mathrm{OH}$ from the active Fenton reaction between $\mathrm{Fe}^{2+}$ of ZIF-Zn-Fe and $\mathrm{H}_{2} \mathrm{O}_{2}$. With the help of the fuel $\left(\mathrm{H}_{2} \mathrm{O}_{2}\right)$, the micromotors will be propelled by the $\mathrm{O}_{2}$ bubbles to accelerate the mass transfer; in addition, the reactive oxygen species (ROS) generated by Fenton reaction can oxidize almost organic compounds in aqueous solution. Herein, in this work, rhodamine $\mathrm{B}(\mathrm{RhB})$ was chosen as the model organic contaminant to study the water treatment efficiency of a Fenton reaction performed by the self-propelled micromotors.

\section{Experimental section}

\subsection{Materials}

Zinc nitrate hexahydrate, silver nitrate, $\mathrm{RhB}, \mathrm{H}_{2} \mathrm{O}_{2}(30 \%, \mathrm{v} / \mathrm{v}$ aqueous solution), ethyl acetate, sodium dodecyl sulfate (SDS), 2-methylimidazole (99\%) was purchased from SigmaAldrich. Polystyrene particles $(38-80 \mu \mathrm{m})$ was purchased from Tianjin Nankai HECHENG S\&T Co., Ltd, China. All analytical reagents were used without further purification, and the water used in all experiments was purified by a Milli-Q system.

\subsection{Synthesis of Ag-ZIF Janus micromotors}

In a typical synthesis, polystyrene@ZIF-Zn-Fe particles were prepared by the following stepwise reaction. A precursor solution was prepared by mixing 2-methylimidazole $(166.0 \mathrm{mg})$, $\mathrm{Zn}\left(\mathrm{NO}_{3}\right)_{2} \cdot 6 \mathrm{H}_{2} \mathrm{O}(500.8 \mathrm{mg})$ and $\mathrm{FeSO}_{4} \cdot 7 \mathrm{H}_{2} \mathrm{O}(93.6 \mathrm{mg})$ in proper amount methanol. The polystyrene particles $(30.0 \mathrm{mg})$ were then added to the precursor solution. The resulting mixture was placed in an oil bath $\left(70^{\circ} \mathrm{C}\right)$ for $10 \mathrm{~min}$. The polystyrene@ZIF$\mathrm{Zn}-\mathrm{Fe}$ microspheres generated during this time were isolated by cooling the reaction mixture to room temperature, collecting the precipitate by free settlement, and washing the precipitate several times with methanol.

The Ag-ZIF Janus micromotors were prepared using polystyrene@ZIF-Zn-Fe microspheres as the base particles. Firstly, the monolayer polystyrene@ZIF-Zn-Fe particles were dispersed initially onto glass slides and coated with $\mathrm{Ag}$ nanoparticles using a Denton Discovery-18 sputter system. The deposition was performed at room temperature with a DC power of $200 \mathrm{~W}$ and an Ar pressure of 2.5 mTorr. In order to obtain a thin film of Ag nanoparticles on the polystyrene@ZIFs-Zn-Fe surface, rotation was turned off and the sample slides were set up at an angle to be parallel to the Ag target, with the deposition time of $20 \mathrm{~s}$. The Ag-ZIF micromotors prepared with uniform Janus half-shell coating were pipette off from the substrate.

\subsection{Materials characterization}

Scanning electron microscope (SEM) images were acquired using a XL 30 ESEM-TMP (Philips Electronics Co., Eindhoven, Netherlands). The energy dispersive X-ray spectroscopy (EDX) spectrum revealed the elements exist in catalysts. Fourier transform infrared (FTIR) spectra were recorded with $\mathrm{KBr}$ pellets on Nicolet 6700 FTIR Spectrometric Analyzer in the range of $4000-500 \mathrm{~cm}^{-1}$. Videos were captured by an optical microscope (YYS-80E, Yiyuan Optical Instrument Co., Ltd, Shanghai).

\subsection{Degradation experiments}

The Ag-ZIF micromotors as catalysts played a dual role leading to high removal rate in this study. Here, RhB removal experiments were performed in a glass Petri dish containing $10 \mathrm{~mL}$ of solution with $\mathrm{RhB}\left(20 \mathrm{mg} \mathrm{L}^{-1}\right), \mathrm{H}_{2} \mathrm{O}_{2}(12 \%)$, SDS (0.2\%) as surfactant, and $5 \mathrm{mg}$ Ag-ZIF micromotors. A small amount of the solution was sampled and immediately centrifuged at a speed of $8000 \mathrm{rpm}$ for $1 \mathrm{~min}$ to remove the solid micromotors, and the RhB concentration in the supernatant was determined by using a UV-visible spectrophotometer (UV-5100B, Metash Instruments (Shanghai) Co., Ltd, China) at its maximum absorbance wavelength of $\lambda=554 \mathrm{~nm}$. All the experimental procedures were conducted under the regular conditions explained above unless otherwise stated.

\section{Results and discussion}

\subsection{Characterization}

The morphology and formation of Ag-ZIF microspheres were verified by SEM and EDX analysis. Fig. 1a shows obviously that spherical morphology with $\mathrm{Ag}$ hemisphere patch (diameter $\sim 80$ $\mu \mathrm{m})$. The EDX measurement is performed in two different positions on the micromotor, which demonstrates that the microparticle is half-coated with Ag. Point 2 (Fig. 1c) is on the side of silver hemisphere, while point 1 (Fig. 1b) is on the other side. As a result, due to the cover of Ag hemispheric layer, almost very few of the Fe and Zn elements are detected on the surface coated with Ag. However, it is observed clearly the presence of $\mathrm{Zn}$ and $\mathrm{Fe}$ on point 1, in agreement with the chemical composition of ZIF-Zn-Fe which indicates that the right hemisphere is polystyrene@ZIF-Zn-Fe composite. The SEM image also illustrates that the resulting Ag layer displays a very rough morphology (Fig. 1a, inset), compared to common Janus particles. ${ }^{16}$ Such close examination of the Ag patch reveals that the $\mathrm{Ag}$ surface is composed of rod-shaped silver nanoparticles with diameters $20-50 \mathrm{~nm}$ and lengths in the range $300-800 \mathrm{~nm}$, which leads to a dramatically increased catalytic surface area and to an improved movement behavior.

To further prove the composition of each sample, the FTIR spectra were recorded for pure ZIF-Zn-Fe, pristine polystyrene particles, polystyrene@ZIF-Zn-Fe microspheres and Ag-ZIF micromotors (Fig. 2). Polystyrene particles are the linear aromatic polymer and are composed of long carbon chains and $\mathrm{C}-\mathrm{C}$ and $\mathrm{C}-\mathrm{H}$ bonds. In the FTIR spectrum of the pure polystyrene particles (Fig. 2a), the peaks at 705 is attributed to the bending vibrations of aromatic ring, and the peaks at 1638 and $2926 \mathrm{~cm}^{-1}$ are assigned to the stretching vibrations of aromatic $\mathrm{C}=\mathrm{C}$ and the symmetric stretching mode of $-\mathrm{CH}_{2}$ in the polystyrene particles. ${ }^{17,18}$ Besides, the bands at $1053 \mathrm{~cm}^{-1}$ are associated with symmetric stretching vibration of $-\mathrm{SO}_{3} \mathrm{H} \cdot{ }^{19,20} \mathrm{As}$ shown in Fig. 2b, the bands in the spectral region of 500$1350 \mathrm{~cm}^{-1}$ and $1350-1700 \mathrm{~cm}^{-1}$ are assigned as the plane 

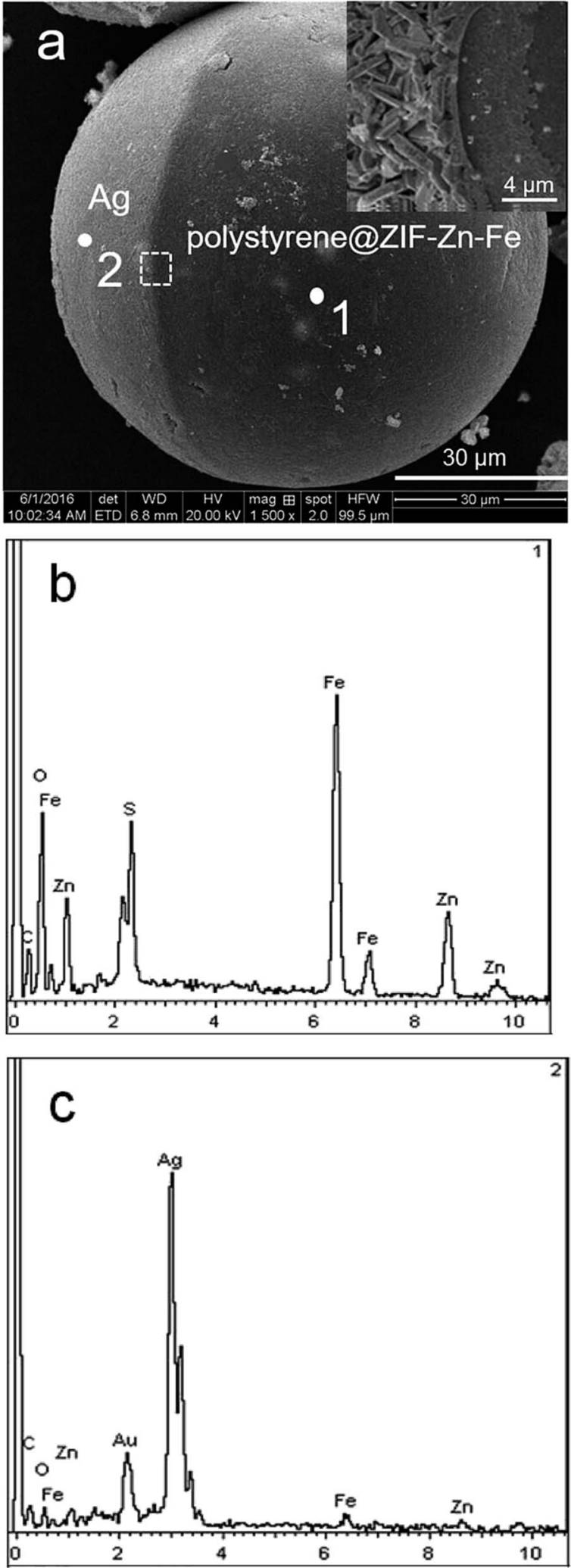

Fig. 1 SEM images (a) of Ag-ZIF microsphere. The scale bar is $30 \mu \mathrm{m}$. The inset highlights the detail in white box in (a). The scale bar is $4 \mu \mathrm{m}$. And EDX spectra (b, c) on point 1 and 2.

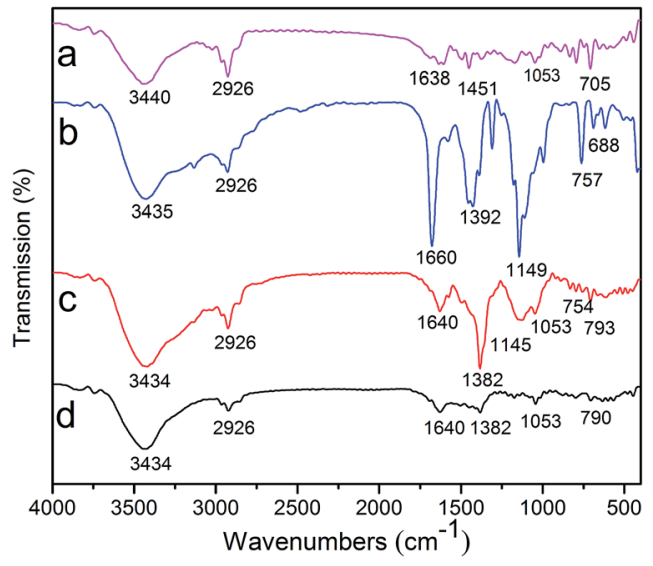

Fig. 2 FTIR spectra of (a) polystyrene particles, (b) pure ZIF-Zn-Fe, (c) polystyreneaZIF-Zn-Fe, and (d) Ag-ZIF Janus micromotors.

bending and stretching of imidazole ring of ZIF-Zn-Fe material, respectively. ${ }^{21}$ Compared to FTIR spectrum of the pure polystyrene particles, the FTIR spectrum of polystyrene@ZIF$\mathrm{Zn}-\mathrm{Fe}$ shows the additional absorption bands at 754, 1145 and $1382 \mathrm{~cm}^{-1}$ of ZIF-Zn-Fe shell (Fig. 2c). It is suggested that the ZIF-Zn-Fe units have successfully grew on the surface of polystyrene particles. The weak peak intensity of absorption bands associated with ZIF-Zn-Fe structure is mainly due to the lower content of ZIF-Zn-Fe material of the core-shell particles. After loading of metallic Ag particles, the resultant Janus micromotors show a low absorption-peak intensity of the functional group, which may be attributed to the existence of Ag in the surface of polystyrene@ZIF-Zn-Fe microspheres (Fig. 2d).

For the propulsion, the Ag-ZIF micromotors were dispersed in the hydrogen peroxide solution. The rough catalytic Ag layer promotes effective bubble evolution and propulsion. To reduce the surface tension and to facilitate bubble ejection from the end of micromotors, $0.2 \%$ of surfactant (SDS) was added into the hydrogen peroxide solution. Fig. 3, and corresponding Video 1 (ESI $\dagger$ ) elucidate the efficient movement of $\mathrm{Ag}$-ZIF micromotors in $6 \% \mathrm{H}_{2} \mathrm{O}_{2}$. These time-lapse images show the ability to simultaneously visualize and track the micromotors, and illustrate that the excellent mobility of micromotors due to the propulsion by bubbling. As we can see, the catalytic Janus micromotor is powered by the decomposition of hydrogen peroxide into molecular oxygen which accumulates in the $\mathrm{Ag}$ patch and eventually gets released from one end of the micromotor as visible bubbles, which led to a directional propulsion thrust. As a result, the average speed of this Janus micromotor exhibiting motion can reach an ultrafast speed of $623 \pm 61 \mu \mathrm{m}$ $\mathrm{s}^{-1}$ in the presence of $6 \% \mathrm{H}_{2} \mathrm{O}_{2}$. Furthermore, once the $\mathrm{Ag}$ - ZIF micromotors are immersed into $\mathrm{H}_{2} \mathrm{O}_{2}$, the continuous and steady generation of bubbles generated from the $\mathrm{Ag}$ hemispheric layer ensures the continuous movement of such micromotors, which is essential for practical environmental applications. It is useful to impart significant mixing during decontamination processes (without external forced convection, e.g., stirring). In addition, the mobility of this $\mathrm{Ag}$-based catalytic micromotors is over $600 \mu \mathrm{m} \mathrm{s}^{-1}$ in $6 \% \mathrm{H}_{2} \mathrm{O}_{2}$, which 


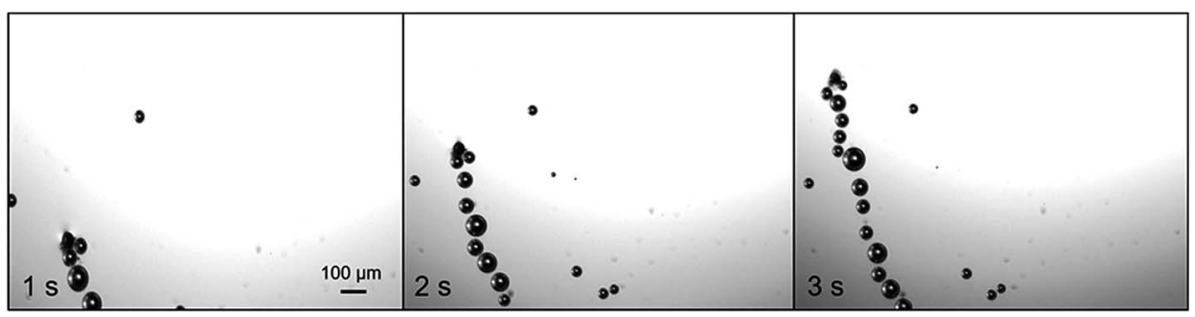

Fig. 3 Motion of self-propelled Ag-ZIF micromotors in $6 \% \mathrm{H}_{2} \mathrm{O}_{2}$ with $0.2 \%$ SDS. Optical snapshots from ESI Video $1 \uparrow$ of micromotors moving at different times.

is quite high compared to that of the Pt-based micromotors that the average velocity is under $15 \mu \mathrm{m} \mathrm{s}^{-1}$ in $6 \% \mathrm{H}_{2} \mathrm{O}_{2} \cdot{ }^{22-25}$ It makes them attractive alternatives to the currently popular Pt-based micromotors for prolonged propulsion in diverse ranges of practical applications.

In order to examine the role of the $\mathrm{H}_{2} \mathrm{O}_{2}$ in the higher propulsion thrust and speed, the self-propulsion of Janus micromotors in different concentrations of peroxide fuel was studied. A certain tiny volume of water solution containing the micromotors was dropped on a glass slide, followed by an equal volume of fuel $\left(\mathrm{H}_{2} \mathrm{O}_{2}\right)$, to get the final concentration of $\mathrm{H}_{2} \mathrm{O}_{2}$ are $1 \%, 3 \%, 6 \%, 9 \%, 12 \%, 15 \%$ and $20 \%$ with $0.2 \%$ SDS, respectively. An optical microscope were used for capturing movies. The speed of the micromotors was calculated using a Tracker software of video analysis. As shown in Fig. 4, the speed of the Janus micromotors increases from $316 \pm 23 \mu \mathrm{m} \mathrm{s}^{-1}$ to $402 \pm 35$ $\mu \mathrm{m} \mathrm{s}^{-1}, 623 \pm 61 \mu \mathrm{m} \mathrm{s}^{-1}, 1299 \pm 98 \mu \mathrm{m} \mathrm{s}^{-1}, 1487 \pm 129 \mu \mathrm{m} \mathrm{s}^{-1}$, $1568 \pm 153 \mu \mathrm{m} \mathrm{s}^{-1}$ and $1650 \pm 160 \mu \mathrm{m} \mathrm{s}^{-1}$ in $1 \%, 3 \%, 6 \%, 9 \%$, $12 \%, 15 \%$ and $20 \%$ fuel, respectively. Apparently, it is observed that the velocity of the Janus micromotors is dependent on the $\mathrm{H}_{2} \mathrm{O}_{2}$ fuel concentration. As expected, the motion of micromotors is accelerated clearly with increasing fuel concentration.

\subsection{Catalytic degradation}

We performed a series of control experiments to validate the property of self-propelled micromotors to remove model contaminants in water. In this work, RhB is chosen as a model

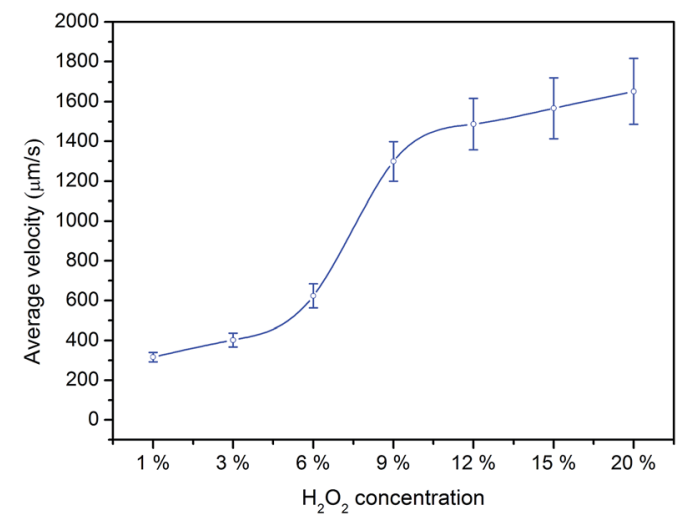

Fig. 4 Effect of the concentration of $\mathrm{H}_{2} \mathrm{O}_{2}$ on the average velocity of $\mathrm{Ag}$-ZIF micromotors $\left(0.2 \%\right.$ SDS concentration, temperature of $25^{\circ} \mathrm{C}$ ). contaminants of xanthene dyes to study the water remediation efficiency performed by prepared bubble-propelled Ag-ZIF micromotors. Based on above discussion, the motion speed of Janus micromotors tends to be moderate when the concentration of $\mathrm{H}_{2} \mathrm{O}_{2}$ reached $12 \%$. Therefore, in experimental processes, we selected this fuel concentration of $12 \%$ as ideal to demonstrate the water purification capabilities of the Janus micromotors, unless otherwise stated. The Ag-ZIF micromotors possess active sites (Fe ions) for heterogeneous Fenton reaction. ${ }^{26}$ The concentration of SDS as surfactant in solution kept $0.2 \%$ to facilitate bubble ejection from the micromotors.

The adsorption and degradation profiles of $\mathrm{RhB}$ against the reaction time under different conditions are shown in Fig. 5. The degradation of $\mathrm{RhB}$ can almost not be observed within 150 min when $\mathrm{H}_{2} \mathrm{O}_{2}$ alone is present, suggesting that the production of oxidizing radicals from $\mathrm{H}_{2} \mathrm{O}_{2}$ could not occur without catalysts. Once involving micromotors, a rapid decrease of the $\mathrm{RhB}$ concentration is observed, revealing that micromotors can efficiently activate $\mathrm{H}_{2} \mathrm{O}_{2}$ to produce ${ }^{\circ} \mathrm{OH}$, resulting in the oxidation removal of organic pollutants in aqueous solution. $\mathrm{RhB}$ removal rate achieves to about $93.1 \%$ within $150 \mathrm{~min}$ in the present of $\mathrm{H}_{2} \mathrm{O}_{2}$ and micromotors. Besides, the Janus micromotors in the absence of SDS are able to oxidize $72.8 \%$ of RhB within 150 min as shown in Fig. 5. SDS can be able to reduce the surface tension and help in the stabilization of bubbles, therefore, the fast bubble generation frequency and the steady motor propulsion can shorten reaction time. Without the addition of $\mathrm{H}_{2} \mathrm{O}_{2}$, the micromotors itself could induce about

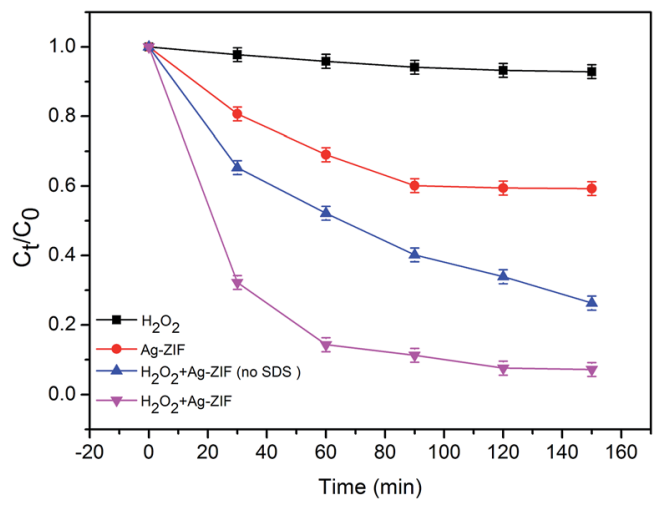

Fig. 5 Removal of RhB under different conditions (initial concentration of RhB, $20 \mathrm{mg} \mathrm{L}^{-1}$; micromotor concentration, $0.5 \mathrm{~g} \mathrm{~L}^{-1} ; \mathrm{H}_{2} \mathrm{O}_{2}$ concentration, $12 \%$; SDS concentration, $0.2 \%$; temperature, $25{ }^{\circ} \mathrm{C}$ ). 
$47 \%$ reduction of $\mathrm{RhB}$ within $150 \mathrm{~min}$, which might be attributed to the surface adsorption of the ZIF-Zn-Fe shell. Therefore, the micromotors not only effectively adsorb the model organic pollutant, but also activate hydrogen peroxide and degrade the adsorbed target.

Further investigation about the effect of $\mathrm{H}_{2} \mathrm{O}_{2}$ concentration on RhB degradation was also conducted. As illustrated in Fig. 6, the degradation rate of $\mathrm{RhB}$ increases with increasing concentration of $\mathrm{H}_{2} \mathrm{O}_{2}$ from $1 \%$ to $12 \%$. About $93.1 \%$ of $\mathrm{RhB}$ decomposed within $150 \mathrm{~min}$ using the self-propelled $\mathrm{Ag}$-ZIF micromotors, as compared to only $58.1 \%$ and $78.8 \%$ using the low concentration of $\mathrm{H}_{2} \mathrm{O}_{2}$ of $1 \%$ and $5 \%$, respectively. Because the motion of the micromotors can be improved with the increasing concentration of $\mathrm{H}_{2} \mathrm{O}_{2}$, the micromotors will be accelerated gradually and, consequently, the mass transfer of the reagents involved in the Fenton oxidation. What's more, as the concentration of $\mathrm{H}_{2} \mathrm{O}_{2}$ increasing, the more ${ }^{\circ} \mathrm{OH}$ is generated in the solution. Therefore, the $\mathrm{H}_{2} \mathrm{O}_{2}$ concentration plays fundamental roles in the degradation processes.

\subsection{Mechanism}

To further clarify the motion of self-propelled micromotors and the process of water remediation, the schematic is shown in Fig. 7. First of all, the high porosity and open pore structure of ZIF-Zn-Fe shell enable fast molecule diffusion of hydrogen peroxide and RhB. In this case, RhB is firstly adsorbed by ZIF$\mathrm{Zn}-\mathrm{Fe}$ in system. As discussed above, it is explained that about $47 \%$ of $\mathrm{RhB}$ is removed only in the presence of the micromotors. Next, ${ }^{\circ} \mathrm{OH}$ is generated through the reaction between $\mathrm{H}_{2} \mathrm{O}_{2}$ and the active catalytic metal sites $\mathrm{Fe}(\mathrm{II})$ of $\mathrm{ZIF}-\mathrm{Zn}-\mathrm{Fe}$. The interactions between $\mathrm{H}_{2} \mathrm{O}_{2}$ and $\mathrm{Fe}$ (II) can be explained by heterogeneous Fenton reaction. Then, the $\mathrm{RhB}$ in aqueous solution can be oxidized and discomposed by the strong oxidizing 'OH radicals. What's more, the Ag-ZIF micromotors mix the fluid vigorously, leading to a convection flow that efficiently diffuses molecules of dye and ${ }^{\circ} \mathrm{OH}$ radicals enhancing the effective degradation of RhB. Therefore,

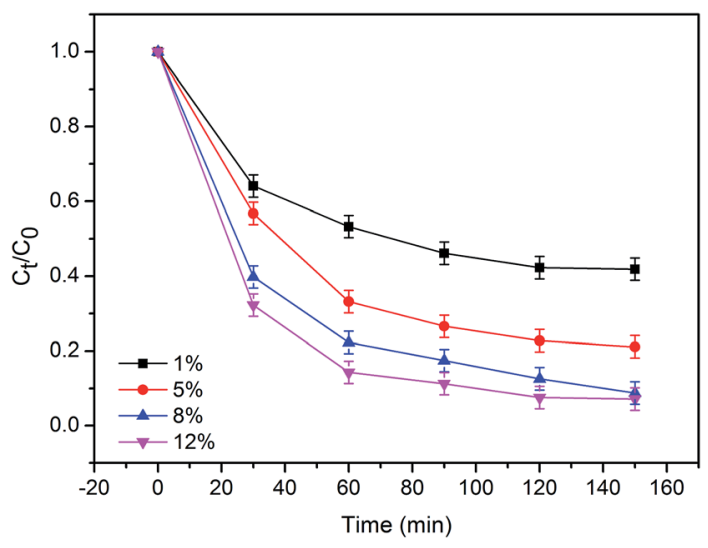

Fig. 6 Effect of the concentration of $\mathrm{H}_{2} \mathrm{O}_{2}$ on the efficiency of removal of RhB (initial concentration of RhB, $20 \mathrm{mg} \mathrm{L}^{-1}$; micromotor concentration, $0.5 \mathrm{~g} \mathrm{~L}^{-1}$; SDS concentration, $0.2 \%$; temperature, $\left.25^{\circ} \mathrm{C}\right)$.

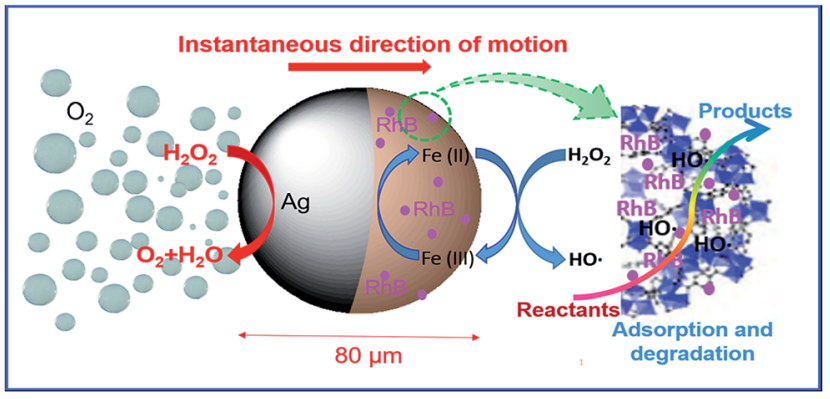

Fig. 7 Proposed mechanism for the removal of RhB by MOFs-based Janus micromotors.

according to the experimental results, the micromotor shows remarkably enhanced catalytic activity for removal of RhB. In one word, in the study of removal of pollutants using micromotors, the adsorption and catalytic sites and the motion of MOFs-based micromotors are responsible for the efficient water purification.

$\mathrm{Ag}$ particles on the surface of micromotors as catalyst enable the decomposition of $\mathrm{H}_{2} \mathrm{O}_{2}$, as a result, oxygen bubbles are generated and released from the side of $\mathrm{Ag}$ hemispheric layer to propel the micromotors. Thus, in this work, $\mathrm{H}_{2} \mathrm{O}_{2}$ acts both as the oxidizing agent in the Fenton reaction and also as the main fuel to propel the Janus micromotors. The decontamination ability of the Ag-ZIF Janus micromotors is associated with their continuous motion, too.

\section{Conclusions}

In summary, we described the preparation of the bubblepropelled MOFs-based Janus micromotors which can be powered efficiently by extremely low concentration of $\mathrm{H}_{2} \mathrm{O}_{2}$. This study also showed that the mobility of these $\mathrm{Ag}$-based catalytic micromotors can be comparable to common Pt-based micromotors. What's more, the speed of the micromotors could be accelerated with increasing the concentration of chemical fuel $\mathrm{H}_{2} \mathrm{O}_{2}$. In addition, thanks to coupling of the high catalytic capacity of MOFs and their autonomous propulsion, the application of the micromotors with highly efficient bubble propulsion in hydrogen peroxide for water purification have shown remarkable removal rate. As aforementioned, a feasible mechanism was proposed to illustrate the corresponding high adsorption rate and catalytic oxidation activity and efficient propulsion of this MOFs-based Janus micromotors. This work is therefore of interest not only for the fundamental science of the Janus micromotors but also for the potential development of multifunctional micromotors for environmental applications and other fields.

\section{Conflicts of interest}

There are no conflicts to declare. 


\section{Acknowledgements}

This work was financially supported by the National Natural Science Foundation, China (Grant No. 51578264), the Shandong Provincial Natural Science Foundation, China (Grant No. ZR2013EEM004), and the Shandong Provincial Science and Technology Development Program, China (Grant No. 2014GSF117008).

\section{Notes and references}

1 S. Tottori, L. Zhang, F. M. Qiu, K. K. Krawczyk, A. FrancoObregón and B. J. Nelson, Adv. Mater., 2012, 24, 811.

2 G. Zhao, T. H. Seah and M. Pumera, Chem.-Eur. J., 2011, 17, 12020.

3 G. Zhao and M. Pumera, RSC Adv., 2013, 3, 3963.

4 H. Wang, G. Zhao and M. Pumera, J. Am. Chem. Soc., 2014, 136, 2719.

5 X. He, Y. K. Bahk and J. Wang, Chemosphere, 2017, 184, 601. 6 O. K. Farha, A. Yazaydın, I. Eryazici, C. D. Malliakas, B. G. Hauser, M. G. Kanatzidis, S. T. Nguyen, R. Q. Snurr and J. T. Hupp, Nat. Chem., 2010, 2, 944.

7 G. Ferey, Chem. Soc. Rev., 2008, 37, 191.

8 G. Lu, S. Li, Z. Guo, O. K. Farha, B. G. Hauser, X. Qi, Y. Wang, X. Wang, S. Han, X. Liu, J. S. DuChene, H. Zhang, Q. Zhang, X. Chen, J. Ma, S. C. J. Loo, W. D. Wei, Y. Yang, J. T. Hupp and F. Huo, Nat. Chem., 2012, 4, 310.

9 E. Haque, J. E. Lee, I. T. Jang, Y. K. Hwang, J.-S. Chang, J. Jegal and S. H. Jhung, J. Hazard. Mater., 2010, 181, 535.

10 H. Lv, H. Zhao, T. Cao, L. Qian, Y. Wang and G. Zhao, J. Mol. Catal. A: Chem., 2015, 400, 81.

11 Y. Pan, Y. Liu, G. Zeng, L. Zhao and Z. Lai, Chem. Commun., 2011, 47, 2071.
12 K. S. Park, Z. Ni, J. Y. Choi, R. Huang, F. J. Uribe-Romo, H. K. Chae, M. O'Keeffe and O. M. Yaghi, Proc. Natl. Acad. Sci. U. S. A., 2006, 103, 10186.

13 K. Kida, M. Okita, K. Fujita, S. Tanaka and Y. Miyake, CrystEngComm, 2013, 15, 1794.

14 X. Fan, W. Wang, W. Li, J. Zhou, B. Wang, J. Zheng and X. Li, ACS Appl. Mater. Interfaces, 2014, 6, 14994.

15 P. Su, H. Xiao, J. Zhao, Y. Yao, Z. Shao, C. Li and Q. Yang, Chem. Mater., 2013, 4, 2941.

16 B. Jurado-Sánchez, S. Sattayasamitsathit, W. Gao, L. Santos, Y. Fedorak, V. V. Singh, J. Orozco, M. Galarnyk and J. Wang, Small, 2014, 11, 499.

17 A. A. Solovev, S. Sanchez, M. Pumera, Y. F. Mei and O. G. Schmidt, Adv. Funct. Mater., 2010, 20, 2430.

18 G. Chen, S. Liu, S. Chen and Z. Qi, Macromol. Chem. Phys., 2001, 202, 1189.

19 H. H. Liu, Q. Y. Chen, S. H. Zhang and X. Y. Li, Chem. Eng. J., 2014, 247, 275.

20 B. Bae, H. Y. Ha and D. Kim, J. Membr. Sci., 2006, 276, 51.

21 Y. Hu, S. Kazemian, S. Rohani, Y. N. Huang and Y. Song, Chem. Commun., 2011, 47, 12694.

22 J. R. Howse, R. A. L. Jones, A. J. Ryan, T. Gough, R. Vafabakhsh and R. Golestanian, Phys. Rev. Lett., 2007, 99, 048102.

23 L. Baraban, D. Makarov, R. Streubel, I. Mönch, D. Grimm, S. Sanchez and O. G. Schmidt, ACS Nano, 2012, 6, 3383.

24 L. Baraban, M. Tasinkevych, M. N. Popescu, S. Sanchez, S. Dietrich and O. G. Schmidt, Soft Matter, 2012, 8, 48.

25 Q. Zhang, R. Dong, X. Chang, B. Ren and Z. Tong, ACS Appl. Mater. Interfaces, 2015, 7, 24585.

26 Venny, S. Gan and H. K. Ng, Chem. Eng. J., 2012, 213, 295. 